\title{
Quantas maneiras existem de se fazer um buraco negro?
}

\author{
Colaboração Ciência Aberta ${ }^{1}$ \\ 4 de Dezembro de 2021
}

\begin{abstract}
Resumo
Este é um artigo de divulgação científica em que discutimos, resumidamente, a partir de [1], quantas maneiras existem de se fazer um buraco negro.
\end{abstract}

palavras-chave: buraco negro, entropia, relatividade geral, divulgação científica

A versão mais atualizada deste artigo está disponível em

https://osf.io/mvb7d/download

https: //zenodo.org/record/5753177

\section{Preâmbulo}

1. Este artigo foi elaborado a partir de [1], seguindo as diretrizes da licença Creative Commons [2].

2. A referência [1], em português, pode ser lida em https://bit.1y/3cUNsWD.

${ }^{1}$ Todos os autores com suas afiliações aparecem no final deste artigo. 


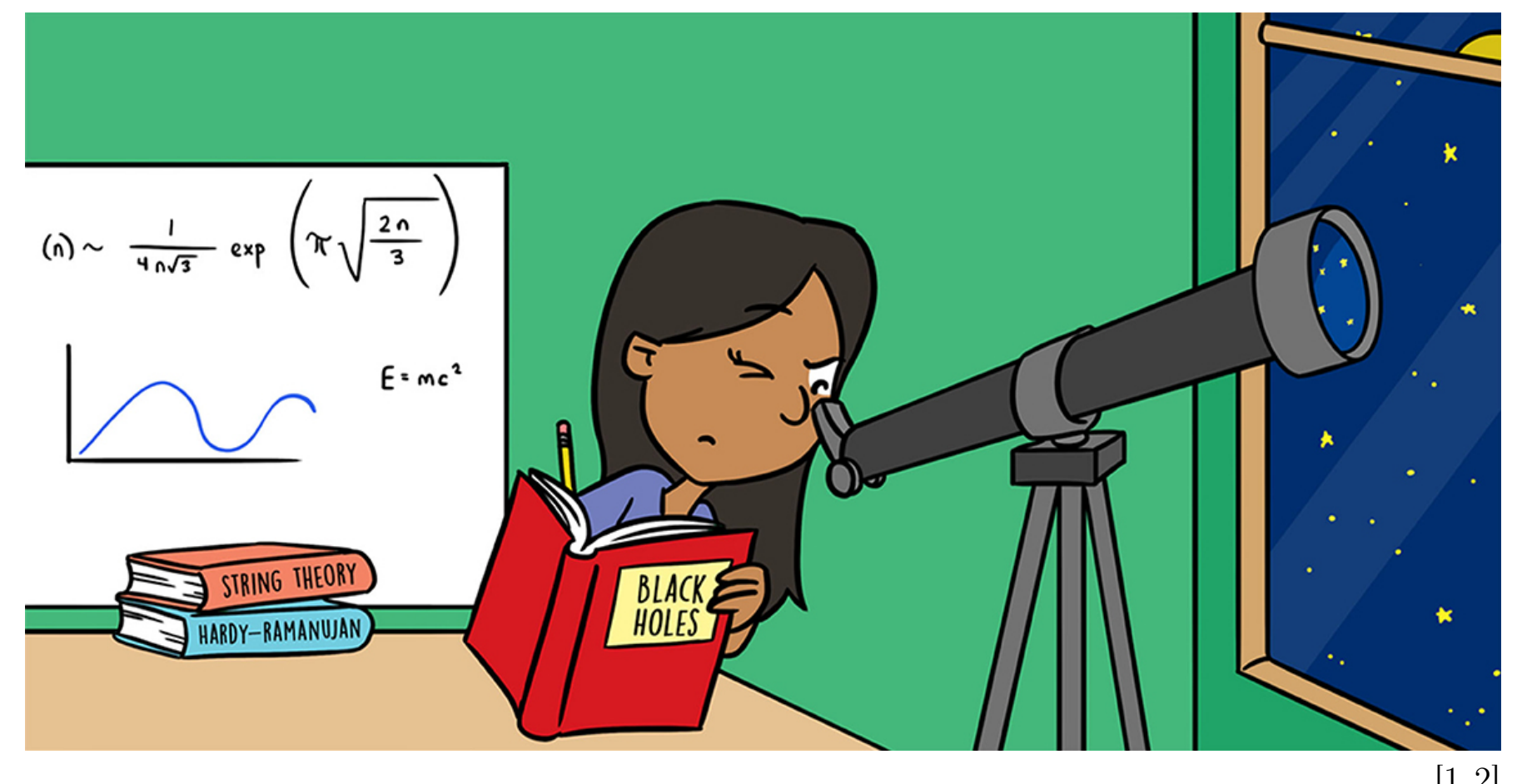

$[1,2]$

\section{Glossário}

3. Amplitude: A amplitude é uma medida da altura dos picos de ondas de água, por exemplo, ou ondas de deslocamento de cordas, ou outros tipos de ondas.

4. Buraco negro: Um buraco negro é um aglomerado de matéria tão densa que nem mesmo a luz consegue escapar de sua superfície ou horizonte de eventos.

5. Entropia: A quantidade de desordem ou arbitrariedade envolvida na especificação dos estados de um determinado sistema físico. A entropia mede o número de maneiras possíveis como isso poderia ser feito.

6. Frequência: Para uma onda (por exemplo, de água ou deslocamento de uma corda), é o número de vezes que a onda atinge sua altura máxima por unidade de tempo. 
7. Horizonte de eventos: A superfície de um buraco negro. Se você está no horizonte de eventos - ou mais perto do centro (isto é, em seu interior) - você não pode escapar de ser sugado, mesmo que seja um raio de luz, que é o objeto que se move mais rápido, segundo a física moderna!

8. Velocidade de escape: É a velocidade mínima necessária para que um objeto consiga escapar da gravidade de um centro de atração (planeta, estrela, etc.).

\section{Introdução}

9. A gravidade é uma força universalmente atrativa, que tenta fazer com que a matéria se aglomere.

10. Isso significa que em diversos sistemas físicos, que vão desde estrelas no final de suas vidas, queimando combustível nuclear, até nuvens de gás que entram em colapso sob o peso de sua própria gravidade, um ponto final natural pode ser o desenvolvimento de um buraco negro.

11. Este é um objeto celeste onde a matéria foi compactada de forma tão densa que mesmo a luz não pode escapar da atração da gravidade em sua superfície, ou horizonte de eventos.

12. Os buracos negros são comuns e misteriosos - nossa própria galáxia, a Via Láctea, hospeda um buraco negro gigante em seu centro!

13. Neste artigo, discutimos os pensamentos atuais sobre um dos mistérios dos buracos negros - como se pode explicar o número de estados físicos distintos (mas semelhantes) que eles escondem atrás de (ou em) seu horizonte de eventos.

14. Os físicos chamam isso de entropia do buraco negro. 


\section{O que é entropia?}

15. Entropia é a quantidade de desordem ou arbitrariedade envolvida na especificação de um determinado sistema físico (ou, grosso modo, o número de maneiras como isso poderia ser feito).

16. Sistemas em estados muito especiais - quase qualquer pedaço de matéria normal em temperaturas muito baixas, ou um quarto muito limpo e organizado, por exemplo - têm baixa entropia.

17. (16) ocorre porque os constituintes do sistema não têm escolha sobre onde estar.

18. Em baixa temperatura, em um pedaço de material, por exemplo, cada átomo fica em um local que minimiza a energia do sistema.

19. Em uma sala muito limpa, todas as peças de roupa são dobradas e empilhadas em uma gaveta.

20. Em contraste, a matéria em alta temperatura, ou uma sala bagunçada, tem alta entropia.

21. Os átomos podem estar se movendo em todas as direções possíveis na matéria a altas temperaturas.

22. Em um quarto bagunçado, camisas e shorts podem ser jogados pela sala à vontade.

23. A entropia de um sistema é definida como o logaritmo do número de configurações possiveis de seus constituintes (átomos para a matéria; camisas e shorts para uma sala bagunçada) que pareceriam praticamente iguais para um observador casual.

24. Para objetos convencionais como mesas, salas ou placas de chumbo, não é surpresa que se possa associar uma entropia ao sistema.

25. Sabemos do que esses sistemas são feitos e como contar as configurações das partes subjacentes. 
26. Para grande surpresa dos físicos teóricos, foi descoberto no início dos anos 1970 por Bekenstein e Hawking, que a essa lista de sistemas comuns com entropia, você pode adicionar buracos negros.

\section{Buracos negros e o insight de Bekenstein}

27. Imagine pegar um pedaço de matéria com uma massa fixa $M$ e esmagálo.

28. A teoria da gravidade de Einstein diz que quando você espreme um objeto massivo em uma região suficientemente pequena do espaço - com um tamanho conhecido como raio de Schwarzschild, $R_{S}(M)$ o objeto se torna um buraco negro.

29. Portanto, seu funcionamento interno permanece um mistério para o mundo exterior, incluindo nós!

30. Se você quiser saber mais detalhadamente o que uma "região pequena o suficiente" significa, o raio de Schwarzschild é dado por

$$
R_{S}(M)=\frac{2 G M}{c^{2}},
$$

onde $G$ é a constante gravitacional e $c$ é a velocidade da luz.

31. O ponto básico é que se você colocar uma quantidade de massa $M$ ou maior em uma esfera de raio menor que $R_{S}(M)$, então ela se tornará um buraco negro.

32. Estamos falando de matéria realmente densa.

33. Para comprimir a Terra de modo que ela se torne um buraco negro, você teria que reduzir seu raio de $6.437 \mathrm{~km}$, aproximadamente, para 9 $\mathrm{mm}$ ! Neste caso, seríamos sugados para o seu horizonte de eventos.

34. A velocidade de escape de um buraco negro é superior à velocidade da luz, ou seja, acima de 1.080.000.000 km/h. A luz não consegue escapar dele e, por isso, ele é chamado de buraco negro. 
35. Felizmente, a natureza nos forneceu exemplos de buracos negros na vida real, então não precisamos nos dar ao trabalho de fazer um.

36. Na verdade, agora você pode até mesmo ver um buraco negro - ou mais precisamente, sua vizinhança.

37. Uma imagem espetacular do buraco negro situado na galáxia M87 - um enorme buraco negro pesando quase 7 bilhões de vezes mais que o nosso Sol - foi recentemente produzida pela colaboração Event Horizon Telescope [3].

38. Jacob Bekenstein ponderou que um observador fora de um buraco negro só pode ver o horizonte de eventos - seu interior está oculto.

39. As propriedades físicas do horizonte de eventos são totalmente caracterizadas por apenas alguns números: no caso mais simples, a massa $M$ do buraco negro.

\section{Questionamentos de Bekenstein}

40. Bekenstein fez os seguintes questionamentos:

(i) Eu não poderia ter formado o buraco negro de massa $M$ de muitas maneiras diferentes?

(ii) Eu poderia tê-lo formado jogando muitos elefantes ou, alternativamente, muitos lêmingues.

(iii) O que aconteceu com as informações sobre como o buraco negro foi formado?

41. Portanto, o buraco negro deve ter uma entropia!

42. O trabalho de Bekenstein - e mais tarde o trabalho mais preciso de Bardeen, Carter e Hawking - conseguiu provar, definitivamente, que os buracos negros realmente têm uma entropia. 
43. E eles foram capazes de produzir uma fórmula para a entropia de um buraco negro em função de sua massa $S(M)$ [4].

44. Mas a natureza precisa dos estados físicos que poderiam explicar $S(M)$ permaneceu misteriosa.

\section{Contando com Hardy e Ramanujan}

45. Suponha que você tenha 5 laranjas e deseja dividi-las entre vários amigos. De quantas maneiras isso é possível?

46. Por exemplo,

$$
5=4+1=3+2=3+1+1=2+2+1=2+1+1+1=1+1+1+1+1 .
$$

47. Vemos que existem 7 maneiras de dividir 5 laranjas: em um grupo de 5, dois grupos de 4 e 1, e assim por diante.

48. Dizemos que o número 5 tem 7 partições, ou seja,

$$
p(5)=7 \text {. }
$$

49. Escrevemos uma função que associa a cada número inteiro o número de partições $p(n)$.

50. Para números $n$ pequenos, como 3,4 ou 5 , calcular $p(n)$ é fácil.

\section{Mas e quanto a $p(\mathbf{1 0 0})$ ?}

52. Se você começar a tentar descobrir isso, verá que os números das partições ficam rapidamente fora de controle!

53. Como devemos determiná-las?

54. Hardy e Ramanujan desenvolveram um truque denominado o método do círculo. 
55. O método do círculo consiste em uma fórmula que permite determinar aproximadamente $p(n)$ e possibilita a criação de um gráfico como o da Fig. 1.

56. No eixo $x$ temos $n$, enquanto que no eixo $y$ temos a precisão da estimativa de $p(n)$.

57. As partições só fazem sentido para números inteiros positivos n, mas sua fórmula fornece uma resposta - e pode ser traçada - para todos os valores de $n$.

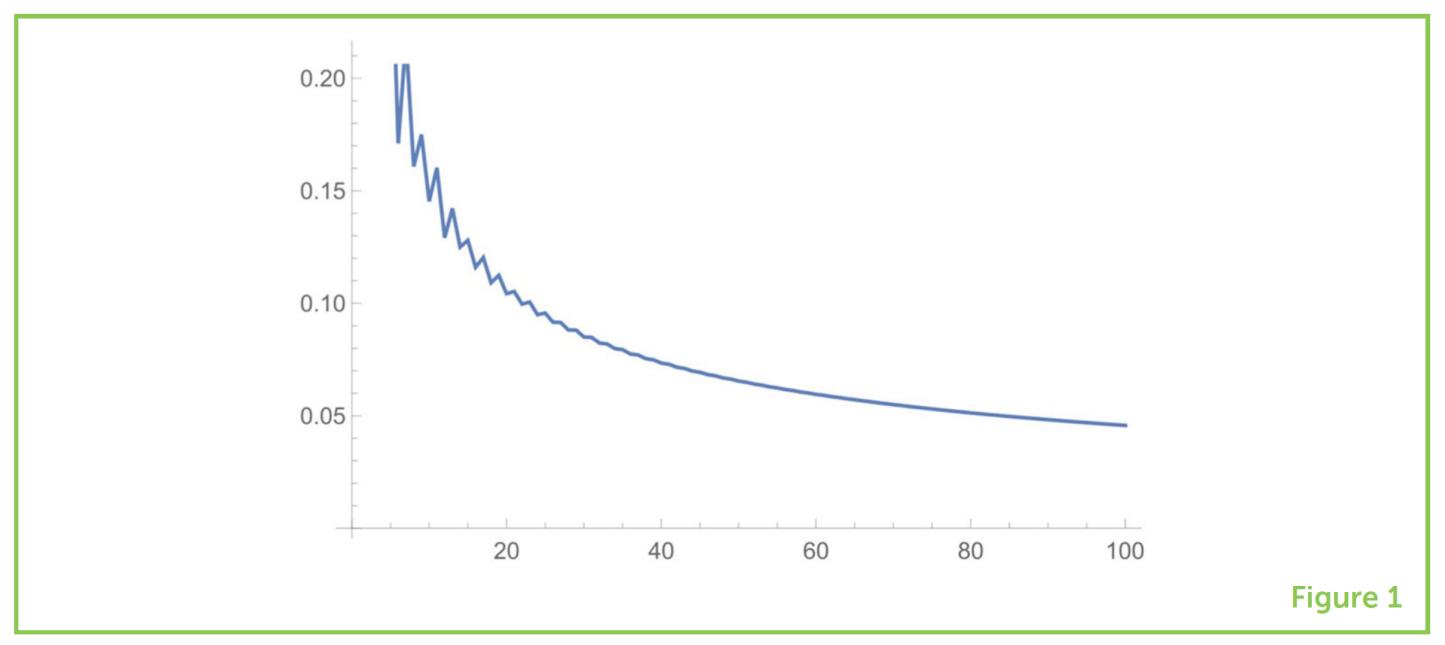

Figura 1: Gráfico da precisão da estimativa de Hardy-Ramanujan de $p(n)$ versus $n$, para valores de $n$ até 100 . O eixo $y$ mostra o erro fracionário da estimativa (então, para $y=0,05$, temos $x=100$, o significa que a fórmula obtém $p(100)$ com uma precisão de cerca de 5\%. Crédito da imagem: John D. Cook $[1,2]$.

\section{E quanto aos buracos negros?}

58. Começamos com os buracos negros e depois desviamos para as partições de inteiros positivos.

59. Como esses dois problemas estão relacionados? 
60. Para colocar esses dois assuntos juntos, primeiro temos que falar sobre um terceiro assunto, chamado teoria das cordas.

61. A teoria das cordas é uma das teorias que os cientistas usam para descrever as menores coisas - ainda muito menores do que os átomos - que compõem o universo.

62. Esses objetos fundamentais são (minúsculos) laços de corda.

63. O estado de menor energia de um minúsculo laço (loop) de corda representaria uma imobilidade perfeita.

64. Você também pode adicionar energia à corda para criar ondas que podem viajar com várias frequências.

65. Na Fig. 2 (onde usamos uma corda de violão ao invés de um loop fechado de corda para facilitar a ilustração), vemos que a onda de frequência mais baixa que você pode colocar na corda tem um pico, enquanto as frequências mais altas (frequentemente chamadas de mais altos harmônicos na música!) têm dois, três ou mais picos.

66. Isso permite várias ondas diferentes possíveis na corda, com frequências fornecidas por números inteiros positivos.

67. É importante ressaltar que também podemos excitar a corda com duas ondas de frequência mais baixa, ou três, ou mais.

68. Aqui, estamos usando o fato de que as energias presentes nas ondas de uma determinada frequência na corda estão sujeitas à mecânica quântica.

69. Mas a mecânica quântica quantifica a energia presente em uma determinada frequência em pedaços discretos - então, temos um número inteiro (como 1, 2, ou 1.729, mas não 2,718 ) vezes a energia associada a uma onda em uma determinada frequência $[5,6]$. 


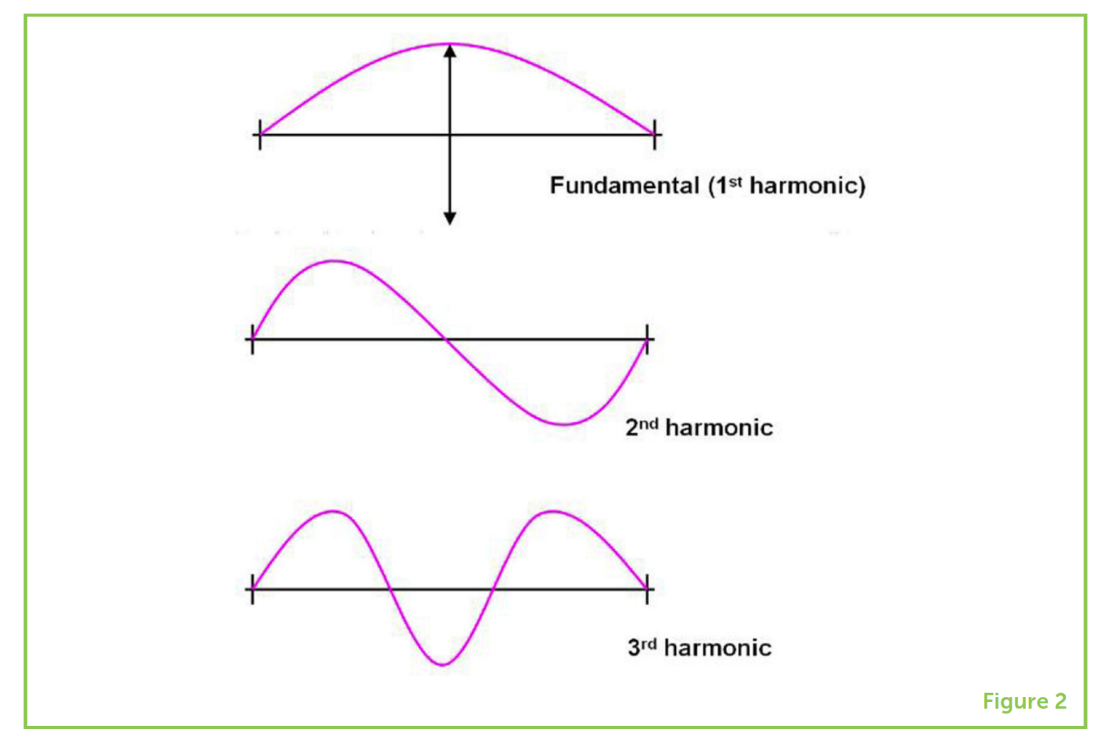

Figura 2: Uma corda de violão clássico permite tocar a frequência mais baixa com qualquer amplitude (e, portanto, colocar qualquer quantidade de energia na corda nessa frequência) $[1,2]$.

70. Agora, estamos a apenas alguns passos de descobrir como calcular a entropia do buraco negro na teoria das cordas!

\section{Contamos com as observações nos itens a seguir.}

72. Poderíamos imaginar ondas com muitas frequências diferentes simultaneamente presentes na corda.

73. Lembre-se de que todas as frequências são dadas por inteiros positivos e queremos controlar o número de "pedaços quânticos" presentes em cada frequência.

74. O custo de energia desses fragmentos quânticos aumenta com a frequência $k$.

75. Frequências mais altas usam mais energia, com a $k$ ésima frequência usando $k$ vezes mais energia que a frequência mais baixa.

76. A equação mais famosa de Einstein nos diz que

$$
E=m c^{2} .
$$


77. (76) significa que a energia da corda vibrante $(E)$ aparece como uma massa $(m)$.

\section{O que concluímos?}

79. Podemos calcular o número de estados de cordas massivas com uma energia $N$ vezes maior do que o modo de energia mais baixa, da seguinte maneira, usando o método de Hardy-Ramanujan, descrito anteriormente.

80. Podemos alcançar o nível de energia $N$ tendo qualquer número de excitações da frequência mais baixa $n(1)$, qualquer número $n(2)$ na segunda frequência mais baixa, qualquer número $n(3)$ com a terceira frequência e assim por diante.

81. Mas, para atingir a energia $N$ total, devemos ter

$$
N=n(1)+2 n(2)+3 n(3)+\ldots
$$

82. Cada escolha do conjunto de números $n(1), n(2)$, etc., que aparece no lado direito de (81) exibe precisamente uma partição de $N$.

83. O número de estados massivos da corda no nível de massa da corda $N$ é dado pelo número de partições de $N$ em inteiros positivos, $p(N)$.

\section{Por que isso está relacionado aos buracos ne- gros?}

84. Uma corda altamente excitada e oscilante na teoria das cordas pode teoricamente ser colocada dentro de seu raio de Schwarzschild e formar um buraco negro. 


\section{Entropia de Bekenstein-Hawking}

85. Como sabemos a massa dos estados das cordas como uma função de $N$ e sabemos o número de tais estados das cordas a partir de $p(N)$, podemos produzir uma fórmula para a entropia $S(M)$.

86. Isso dá uma previsão (correta) para a entropia de buracos negros em termos de números de partição [7], dada por

$$
S(M)=\frac{k_{B} A(M)}{4 l_{P}^{2}},
$$

onde $k_{B}$ é a constante de Boltzmann, $A(M)$ é a área do buraco negro e $l_{P}$ é o comprimento de Planck.

\section{Considerações Finais}

87. A entropia mede o número de (micro)estados de um sistema físico.

88. Note que a entropia do buraco negro, em (86), depende exclusivamente de sua área, já que os demais termos são constantes.

89. Com base nisso, o que poderíamos dizer sobre o interior de um buraco negro? [8] Estaria toda sua massa em seu interior ou, talvez, em sua superfície (horizonte de eventos)? [9, 10]

\section{Ciência Aberta}

$\mathrm{O}$ arquivo latex para este artigo, juntamente com outros arquivos suplementares, estão disponíveis em [11]. Seja coautor(a) deste artigo, envie sua contribuição para mplobo@uft. edu.br.

\section{Consentimento}

Os autores concordam com [12]. 


\section{Como citar este artigo?}

https://doi.org/10.31219/osf .io/mvb7d https://zenodo.org/record/5753177

\section{Licença}

CC-By Attribution 4.0 International [2]

\section{Referências}

[1] Kachru S (2020) "How Many Ways Are There to Make a Black Hole?." Front. Young Minds. 8:467994.

https://doi.org/10.3389/frym.2020.467994

[2] CC. Creative Commons. Attribution 4.0 International (CC BY 4.0) https://creativecommons .org/licenses/by/4.0

[3] Event Horizon Telescope. https://eventhorizontelescope.org

[4] Hawking, S. 1998. A Brief History of Time. New York, NY: Bantam.

[5] Lobo, Matheus P. "On the Wave-particle Duality: Mass and Frequency." OSF Preprints, 18 May 2019.

https://doi.org/10.31219/osf.io/9xa6v

[6] Lobo, Matheus P. "The Insight Behind the Discovery of Quanta by Max Planck." OSF Preprints, 19 May 2019.

https://doi.org/10.31219/osf .io/a9xmt

[7] Susskind, L. 2009. The Black Hole War. New York, NY: Back Bay Books.

[8] Lobo, Matheus P. "The Interior of a Black Hole and the Void of Spacetime." OSF Preprints, 12 May 2019.

https://doi.org/10.31219/osf.io/awfx8 
[9] Lobo, Matheus P. "A Hole in the Black Hole." OSF Preprints, 18 Apr. 2019. https://doi.org/10.31219/osf.io/js7rf

[10] Lobo, Matheus P. "Hollow Black Holes." OSF Preprints, 12 Aug. 2019. https://doi.org/10.31219/osf .io/rewub

[11] Lobo, Matheus P. "Open Journal of Mathematics and Physics (OJMP)." OSF, 21 Apr. 2020.

https://doi.org/10.17605/osf .io/6hzyp

[12] Lobo, Matheus P. "Simple Guidelines for Authors: Open Journal of Mathematics and Physics." OSF Preprints, 15 Nov. 2019. https://doi.org/10.31219/osf .io/fk836

\section{Colaboração Ciência Aberta}

\section{Viviane Vitória Machado Mariano ${ }^{1}$}

(autora principal, vvmmvitoria@gmail.com)

Victor Manoel Soares da Conceição1,2

Matheus Pereira Lobo ${ }^{1,2}$

https://orcid.org/0000-0003-4554-1372

Pâmella Gonçalves Barreto Troncão ${ }^{1}$

https://orcid.org/0000-0002-6340-5983

${ }^{1}$ Universidade Federal do Tocantins (Brasil)

${ }^{2}$ Universidade Aberta (UAb, Portugal) 